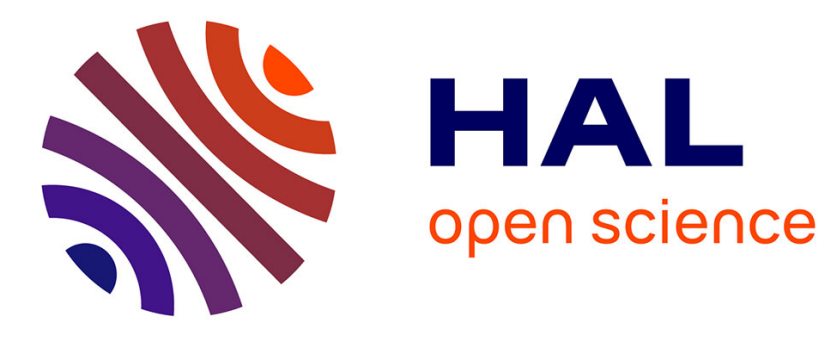

\title{
Multiple constrained QoS routing with RPL
}

Walid Khallef, Miklós Molnár, Abderrahim Benslimane, Sylvain Durand

\section{To cite this version:}

Walid Khallef, Miklós Molnár, Abderrahim Benslimane, Sylvain Durand. Multiple constrained QoS routing with RPL. ICC: International Conference on Communications, May 2017, Paris, France. 10.1109/ICC.2017.7997081 . lirmm-01584511

\section{HAL Id: lirmm-01584511 https://hal-lirmm.ccsd.cnrs.fr/lirmm-01584511}

Submitted on 14 Apr 2021

HAL is a multi-disciplinary open access archive for the deposit and dissemination of scientific research documents, whether they are published or not. The documents may come from teaching and research institutions in France or abroad, or from public or private research centers.
L'archive ouverte pluridisciplinaire HAL, est destinée au dépôt et à la diffusion de documents scientifiques de niveau recherche, publiés ou non, émanant des établissements d'enseignement et de recherche français ou étrangers, des laboratoires publics ou privés. 


\title{
Multiple Constrained QoS Routing with RPL
}

\author{
Walid Khallef, Miklos Molnar, Abderrahim Benslimane, Sylvain Durand \\ University of Montpellier, Montpellier, France \\ Email: (walid.khallef)(Miklos.Molnar)@lirmm.fr \\ Universit d'Avignon et des Pays de Vaucluse, Avignon, France \\ Email: abderrahim.benslimane@univ-avignon.fr \\ University Paul-Valery, Montpellier, France \\ Email: sylvain.durand@lirmm.fr
}

\begin{abstract}
In recent years, there have been significant efforts to standardize a routing protocol for Low-power and Lossy Networks (LLNs). This effort has culminated in standard IPv6 routing protocol for LLNs (RPL). The main interest of RPL is to improve routing in an LLN minimizing the usage of network resources. For this, RPL builds acyclic graphs and applies an Objective Function (OF) which is responsible of choosing the preferred parent and the best links during the construction of the Destination Oriented Directed Acyclic Graph (DODAG). This paper introduces a new OF, based on a Non-Linear Length (NL-OF) which takes into account any number of metrics and constraints for QoS routing. NL-OF ensures that each path in the DODAG respects the input constraints. The NL-OF can be used to meet the requirements of sensible applications, such as real-time applications. A significant part of this work aims at studying the theoretical aspect of the NL-OF. Finally, using Cooja simulator, we evaluate the performance of NL-OF. We show that our new Objective Function gives a good result and outperforms the three existing OFs when considering three QoS parameters which are end-to-end Delay, Packet Loss and Jitter.
\end{abstract}

\section{INTRODUCTION}

Low-power and Lossy Networks (LLNs) are composed of a large population of constraint devices. These devices have to be connected together using lossy links which impose a challenging routing task. The most widely adopted standard for routing is IPv6 routing protocol for LLNs (RPL), which is specified in [1] by ROLL working group of IETF. The birth of RPL opened interesting research fields which have witnessed a large body of works on routing problems. Despite the efforts to improve routing by RPL, routing with Quality of Service (QoS) has been somewhat overlooked.

In this paper, we investigate to construct a routing scheme for QoS in RPL during the construction of the routes. RPL selects nodes and optimize routes within an RPL instance using an Objective Function (OF) [2] that defines how the route will be constructed. There are three predefined OFs in RPL (OF0, MRHOF with ETX and MRHOF with energy) [2], [10]. All of them try to construct a Directed Acyclic Graph (DAG) with respect to specific metrics. OF0 constructs a DAG with the lowest number of hops. MRHOF-ETX constructs a DAG with the lowest Expected Transmission Count (ETX) along the path. MRHOF-energy minimizes the energy consumption. However, some applications require several constraints of QoS to operate correctly.
In order to construct a constrained DAG (a DAG that respects QoS constraints), we design a new objective function that uses the non-linear length concept (NL-OF), which is an efficient concept to calculate the paths subject to multiconstraints [5]. NL-OF takes into account an arbitrary number of metrics and provides a path obeying all input constraints.

The existing objective functions take into account only one metric [2], [10] or combine two metrics [11] in an additive or lexical manner. As a result the DAGs cannot fully satisfy some recent applications which require several QoS constraints at once (errors, loss and delay, etc.). For instance, the hop count routing metric allows choosing the shortest path, but it does not necessarily ensure the end-to-end delay requirement, which is an important constraint for interactive applications. A path with low delay does not always guarantee a desired minimal packet loss. An interesting OF was proposed in [9] to combine a set of metrics using Fuzzy Logic (Objective Function for RPL based on Fuzzy Logic or OF-FL). This OFFL uses linguistic variables to describe the state of each metric separately. The linguistic variables are between true and false and each QoS metric is associated to some variables ("near", "vicinity" and "far" for hop count for example). Then the membership function collects the information to quantify the linguistic terms which are largely used in Fuzzy Logic system. The proposed OF-FL is very interesting when the goal is to optimize globally the network. In other words, when there are no defined constraints to respect. Unfortunately, it is not the case with some recent applications having strict requirements (e.g. the end-to-end delay must be less than $30 \mathrm{~ms}$ ). Therefore, using the combination of metrics does not guarantee that each path respects the end-to-end constraints from the root to any node. To improve these existing methods we propose NL-OF which satisfies the end-to-end constraints and provides a DAG that meets the requirements of applications relying on QoS. NL-OF and OF-FL cannot be compared objectively because the test environment cannot be the same, NL-OF seeks a DAG respecting a set of input constraints while OF-FL tries to find a DAG with equal optimisation of metrics without defined constraints.

The remainder of this paper is organized as follows. In Section II we give a brief overview about RPL and its routing metrics. Section III introduces problem formulation. Section IV presents the routing with QoS constraints and the limitation 
of the DAG structure. In Section V, we present our NLOF. Section VI presents numerical results and Section VII concludes this paper.

\section{RPL OVERVIEW}

RPL [1] is an IPv6 Routing Protocol for Low-power and Lossy Networks. Since the LLNs do not have predefined topologies, RPL has to discover links and then select peers sparingly in such a way that no cycles are present. To this end, a Directed Acyclic Graph (DAG) built according to one or more Destination Oriented DAGs (DODAGs), one DODAG per root, using an Objective Function (OF) [2] to reach specific objectives.

We can divide the nodes in RPL in three types. The first type is the root node often corresponding to a Border Router, which is frequently the destination of the communications. It starts the DODAG construction by sending DODAG Information Object (DIO) messages. Each node that receives the message from several potential parents runs an algorithm to choose an appropriate parent. The choice is based on a metric and constraints defined by the Objective Function OF. The second type are routers, that repeat the same steps and the process terminates when a DIO message reaches the third type of nodes (the leaves) or when no more nodes are left in range.

\section{A. The Objective Function}

During the construction of the DODAG, RPL uses an Objective Function [2], which specifies how the routing metrics and constraints [3] are taken into account to reach the desired DODAG. There are three predefined OFs in RPL (OF0, MRHOF with ETX and MRHOF with energy) [2] [10]. All of them try to construct a Directed Acyclic Graph (a set of DODAGs) with respect to specific metrics. OF0 produces a DAG with the lowest number of hops. MRHOF-ETX constructs a DAG with the lowest Expected Transmission Count (ETX) along the path. MRHOF-energy minimizes the energy consumption. However, some applications such as video on demand and collaborative applications, require several constraints of QoS to operate correctly. RPL implementations can choose to adopt a simple approach based on the use of a single metric with no constraint (the optimization of the cost for example). Whereas other implementations can use a larger set of link (and/or node) routing metrics and constraints. We are interested in the optimization of the cost respecting several Quality of Service (QoS) constraints.

The result of the route construction is a Directed Acyclic Graph (DAG) with respect to the specific metrics as the hopcount, the energy consumption or the expected transmission count [2] [10].

RPL can separate the OFs from the core of the protocol which allows it to meet the different optimization criteria required. Each DODAG instance in RPL's DAG is associated with a particular $\mathrm{OF}$.

\section{B. RPL routing attributes (Metrics and Constraints)}

Unlike traditional wired networks, LLNs have unique characteristics that require the specification of new routing metrics and constraints [3]. There are several metrics and constraints based on them, which can be used in path computation by RPL and can be categorized into two basic types:

- Node metrics and related constraints (e.g., node state and attribute and node energy.).

- Link metrics and related constraints (e.g., link throughput and link latency.).

\section{QoS metrics in RPL}

Before presenting our proposition, let us give a brief overview about the most interesting node and link metrics for our study on QoS routing in RPL [3].

- Hop count: metric to report the number of traversed nodes before reaching a given destination.

- Node energy: an important metric which permits to avoid selecting a node with low residual energy.

- Latency: this metrics allows to adjust globally the endto-end delay on the subnet, on a link-by-link basis, or not at all. The minimization of this metric is critical fo real time applications.

- Link reliability: as the change in link quality can affect network connectivity, the reliability of links is crucial. Different link reliability metrics to reflect several reliability aspects (link quality level, ETX metric, etc.) can be defined.

- Moreover, additional metrics can be proposed for QoS aware applications such as the jitter and the probability of packet losses.

So, our work is based on link metrics. In the following section we formulate the multi-constrained routing problem.

\section{Problem Formulation FOR the DODAG COMPUTATION WITH QOS}

Let $G=(N, E)$ be the weighted connected graph representing the topology, where $N$ is the set of nodes (devices) and $E$ is the set of edges representing the possible links (Figure. 1.A). Each edge $e$ is associated with $M$ weights corresponding to the QoS metrics (given by a weight vector $\left.\vec{w}(e)=\left[w_{1}(e), w_{2}(e), \ldots, w_{M}(e)\right]^{T}\right)$ and $c(e)$ is the cost of using the link. We suppose that the link values are the same in both directions.

The objective is to build a DODAG that connects the root node $s$ (a target Border Router for example) to the others nodes (routers and leafs) with the guarantee that each path between the root and the leaves (represented by the set $D$ ) respects the end-to-end constraints given by the constraint vector $\vec{L}=$ $\left[L_{1}, L_{2}, \ldots, L_{M}\right]^{T}$. These paths can be used in both directions. So, let us denoted the path from $s$ to $d_{j}$ by $p_{d_{j}}$ (all examined paths in the paper have $s$ as origin).

In LLNs the link and node metrics are usually (but not always) additive. In this work we only consider additive 
metrics ${ }^{1}$. For additive metrics, the weight of a path $p_{d_{j}}$ corresponding to the metric $i$ is given by:

$$
w_{i}\left(p_{d_{j}}\right)=\sum_{e \in p_{d_{j}}} w_{i}(e) .
$$

The final DODAG (cf. Fig. 1.B) has to contain a feasible path from the root to each leaf such that:

$$
w_{i}\left(p_{d_{j}}\right) \leq L_{i}, i=1, \ldots, M, j=1, \ldots,|D|
$$

We can consider two problems:

- Multi-Constrained DODAG problem, where we are interested by feasible paths satisfying the constraints

- Multi-Constrained Minimum Cost DODAG problem, if we also take into account the cost optimization. This latter can be the cost of paths or the cost of the DODAG

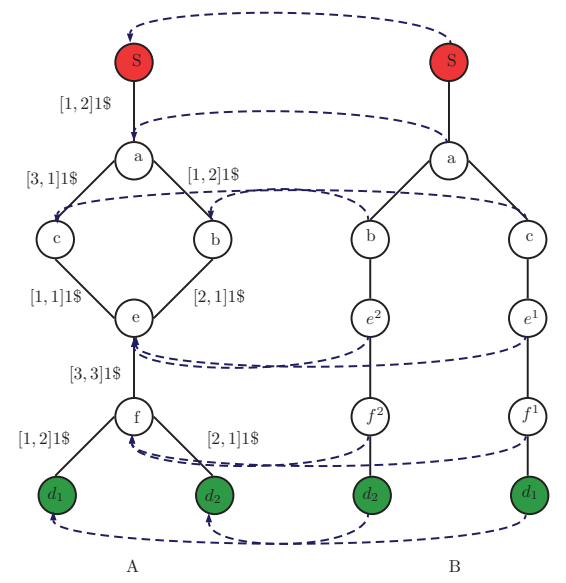

Fig. 1. Example of LLNs topology and its DAG.

Note that, unlike the previous works, we propose to handle an arbitrary number of metrics and constraints to satisfy QoS routing requirements. Our objective is the minimization of the cost respecting the QoS constraints. One of the most challenging research tasks is the routing with multiple constraints (i.e., finding a unicast route or multicast route or even broadcast that respects a set of QoS constraints). A route (even if it is a simple path) can be good for a given metric but bad for another. The different constraints on the different metrics can leave to severe contradictions. In this section we propose the analysis of DAGs proposed in RPL and highlight some limitations in the case of multi-constrained QoS routing.

\section{A. Limitation of the DAGs}

As described above, the DAG is a tree-like structure. It is designed to connect the root node with other nodes specifying by an OF. For data forwarding, each node has only one parent node.

Figure 1 shows the limitation of using a DAG proposed in RPL with multiple QoS constraints. As shown there are two

\footnotetext{
${ }^{1}$ Bottleneck metrics can be easily dealt by pruning from the graph all the links that do not satisfy the value of bottleneck. The multiplicative metrics can be transformed into additive metrics by using a logarithm function.
}

feasible paths from the route node to the leafs nodes, which are $\left(\mathrm{S}-\mathrm{a}-\mathrm{c}-\mathrm{e}-\mathrm{f}-d_{1}\right)$ and $\left(\mathrm{S}-\mathrm{a}-\mathrm{b}-\mathrm{e}-\mathrm{f}-d_{2}\right)$ with respect the end-to-end constraints $\vec{L}=[9,9]^{T}$. The routes are not interchangeable, the path (S-a-b-e) can not be replaced by (S-a-c-e) without the violation of the QoS constraints for the destination $d_{2}$ and inversely, (S-a-c-e) can not be replaced by (S-a-b-e) for $d_{1}$. Using a simple DODAG in RPL, the nodes $e$ and $f$ cannot be used twice and $e$ can not have two parents; there is no feasible DODAG.

Trivially, in the solution of this critical situation, nodes and links should be visited several times, preserving the destination oriented tree-like property of the structure.

Despite the limitations of DODAGs proposed by the protocol, in this work we keep their construction as it is proposed in RPL. This is to maintain the route construction mechanism and to only focus on the OF for QoS routing.

\section{PROPOSITION}

To select routes, RPL uses an Objective Function (OF0), which takes into account only one metric. OF0 remains insufficient to build a DAG (or DODAGs) that can satisfy more precise QoS requirements. Some works have been done to ensure the QoS in RPL, they try to combine two metrics or more using different methods. We propose a solution for the QoS routing with RPL by extending its mechanism to support several metrics and QoS constraints. Our DODAG construction is based on an earlier introduced non-linear length of paths using multiple metrics [5].

\section{A. Non-Linear Length based Objective Function}

Remember each link is associated with an $M$-dimensional weight vector $\vec{w}(e)$ and the QoS requirement is given by $\vec{L}$. Since the metrics are additional, an end-to-end weight vector $\vec{w}\left(p_{d}\right)=\sum_{e \in p_{d}} \vec{w}(e)$ can be associated to path $p_{d}$, which is the vector sum of the link weights along this path according to Equation 2. The quality of a path $p_{d}$ from the source $s$ to the node $d$ can be measured by the following non-linear length [5]:

$$
l\left(p_{d}\right)=\max _{i=1, \ldots, m}\left(\frac{\sum w_{i}(e)_{e \in p_{d}}}{L_{i}}\right)
$$

This length function permits to normalize the constraints and take into account the most critical one of a path, which allow us to verify the feasibility of a given path regarding all constraints simultaneously. If the non-linear length of a path $l\left(p_{d}\right) \leq 1$, the path is feasible for all constraints.

Our objective (NL-OF) is to construct DODAGs from roots to nodes such that the non linear length is the smallest possible. Trivially, a constructed instance will depend on the set of roots, the set of metrics and the QoS requirement $\vec{L}$.

\section{B. Constructing DODAGs with the NL-OF}

Let us assume that there is a single root node and that each other node can store the weight vector computed from the root to this node.

As already pointed out, the construction of the DODAG starts from the root which sends a DIO message to its 
neighbors. We propose the DIO message contains the values of the cumulated weight vector from the root (the root has a null vector for the metrics). After receiving all possible DIO messages in RPL, a node $v$ can compute the cumulated weight vector for each path concerned by the messages. Let $\vec{w}\left(p_{u}\right)$ be the weight vector for the node $u$, which send a DIO message to node $v$ and let $\vec{w}(e)$ the weight on the link $e$ between $u$ to $v$. Trivially, the weight vector $\vec{w}\left(p_{v}^{u}\right)$ associated with the path from the source to $v$ passing by $u$ is:

$$
\vec{w}\left(p_{v}^{u}\right)=\vec{w}\left(p_{u}\right)+\vec{w}(e)
$$

$v$ can select all parent nodes, which are in feasible paths (with non-linear length less then 1) and choose its preferred parent (Figure 2). The preferred parent is the node, which insure the minimal non-linear length for $v$. If a node cannot compute all metrics for the path through a neighbor node $u$, the non-linear length of this path will be set to infinity. Subsequently, the node $v$ sends a DIO message to its neighbors if its non-linear length on the preferred path is less than 1 and so forth until reach the other nodes. The first range of nodes (root's neighbors) will choose the root as a preferred parent and send the DIO message to their neighbors, etc.

Our algorithm select parent nodes and construct acyclic graphs in a greedy manner (Figure 2.B). However, the optimal paths with minimal non-linear length are not guaranteed by this procedure. In other words, the constructed DODAG does not always contain the paths with minimum length, and some feasible paths may be lost (e.g. $d_{2}$ in Figure 2.B).

\section{Analysis of the solution}

To illustrate the weakness of the greedy strategy, let us analyze the exact solution of our optimal QoS routing problem.

1) Exact solution: if our objective is the minimization of the non-linear length from the source to each node, the exact solution (set of routes, which contains the best paths) is not a tree. Seeking an exact solution does not produce necessarily a DAG as a route. As it is mentioned in [6] a sub-path of a shortest path using the non-linear length is not necessarily a shortest paths. Hence, even if the preferred parent has the shortest sub-path from the root, it does not guarantee that using this sub-path will lead to the path with the best non-linear length, to select the path with minimal non-linear length. In the worst case, all paths from the source to the node should be compared. (As an illustration, there is two feasible paths to the node $e$ in Figure. 2). The number of paths can grow in an exponential manner even if we can reduce the research space using the Pareto dominance [8]. A path $p_{d}$ dominates another path $p_{d}^{\prime}$ if: $l_{i}\left(p_{d}\right) \leq l_{i}\left(p_{d}^{\prime}\right), i=1, \ldots, m$. For example the path $\{s, b, f\}$ dominates the path $\{s, a, f\}$ in Figure 2 .

2) Approximate solution: Since RPL is designed for LLNs in which the devices are constrained with memory, computation capacity and energy, the storage of a large number of paths and the computation of the exact solution is very costly and not always feasible. So we use a simple greedy method for the construction of a DAG (as we explained above). We propose a DAG which is an approximate solution since all the possible and feasible paths are not stored and transmitted for further computation.

To summarize: Adding all non-dominated paths to our OFNL mechanism, one can ensure that all possible sub-path are preserved. This gives to each successor the opportunity of choosing the best path according to the objective. Notice that when the exact solution is computed, some overlap between paths may be produce (cf. Figure 1).

\section{Complexity}

As mentioned in [1], the rate of DIO transmission is controlled by Trickle algorithm ${ }^{2}$, so the number of DIO messages does not change when we use NL-OF.

Let $|N|$ be the number of nodes and $|E|$ the number of edges, each node saves one DIO message containing the vector sum from the border router. Selecting the minimum non-linear path length among $V$ different path lengths takes at most $\log (V)$. Calculating the length from a given node to the border router takes $\theta(m)$ when there are $m$ metrics while verifying the feasibility condition takes $\theta(m)$ at most. The worst-case time-complexity of the greedy solution is

$$
\theta(V \log (V)+m|E|)
$$

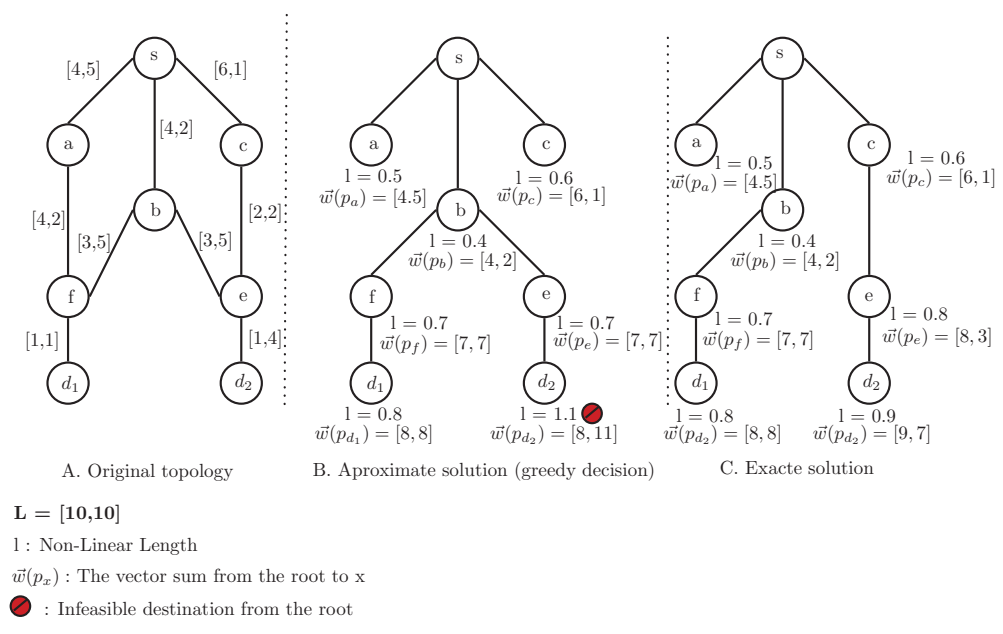

Fig. 2. Non-dominated path

\section{Performance Evaluation}

In this section, we conduct numerical studies to compare our proposition with three OFs predefined in RPL. OFO uses only the hop count, while MRHOF with ETX minimizes the Expected Transmission Count along the path and MRHOF with energy chooses the DAG's nodes with minimum energy consumption. The first part is dedicated to evaluate the performance of NL-OF, MRHOF-ETX, MRHOF-energy and OF0 in term of three QoS metrics of interest: End-to-End Delay, Packet Loss Ratio and Jitter. The second part will evaluate RPL in term of Average Power Consumption. In order to

\footnotetext{
${ }^{2}$ The Trickle algorithm controls the amount of routing traffic in the form of DIO's that enter the network.
} 
better understand our simulation, we present in what follows the explanation of each performance metric and the simulation environment.

\section{A. Performance metrics}

1) QoS performance metrics: The first performance metric is the End-to-End Delay. It refers to the time taken for a packet to be transmitted across a network from a node to the DAG root. The second performance metric is the Packet Loss Ratio, which measures the ratio of packets lost to the total packets sent. The third one is the delay that varies over time i.e. Jitter.

2) RPL performance metric: The key metric for RPL evaluation is the Average Power Consumption which is the average percentage of power consumed by all the nodes in the network.

\section{B. Network setup}

To analyse the results with high level of accuracy, we use Cooja simulator under Contiki operating system [12]. We design in all simulations a network of 39 routers and 1 root of sky node type. The nodes are randomly distributed over a square space $(100 \mathrm{~m} \times 100 \mathrm{~m})$. For the control traffic, we define the DIO Interval Minimum which is used as an initial interval for the control packet transmission and DIO Interval Doublings ${ }^{3}$ to place an upper limit on the rate of this transmission. The start delay defines when the nodes start transmitting their messages to the sink node. To reach a stable state we run each simulation 1800 s. Table I presents the simulation parameters.

TABLE I

PERFORMANCE PARAMETERS

\begin{tabular}{|l|l|}
\hline Parameters & Values \\
\hline Square Space & $(100 \mathrm{~m} \times 100 \mathrm{~m})$ \\
RPL Nodes & 40 \\
DIO Min & 10 \\
DIO Doubling & 70 \\
Type of Nodes & Sky \\
Radio Environment & Unit Disk Graph \\
& Model (UDGM) \\
Start Delay & $60 \mathrm{~s}$ \\
Simulation Time & $1800 \mathrm{~s}$ \\
\hline
\end{tabular}

Notices: we fixed the end-to-end constraints as follows. MAX-END-TO-END-DELAY $=70$ and MAX-PACKETLOSS $=70$. So, $L=[70,70]$. All information about Delay, Packet Loss and Jitter are gathered using data-collected-view option in Cooja, which presents the simulation results in graphical manner and statistic manner.

We only consider the communication between nodes and the root, local communication is outside of scope of this paper.

\footnotetext{
${ }^{3}$ DIO Interval Minimum and DIO Interval Doubling present the Trickle's parameters [14]. In RPL each node use Trickle's parameters to control the rate of DIO transmission.
}

\section{Simulation and discussion}

The aim of the first part of the simulation is to analyse the response of OFs to the requirements of susceptible applications, such as control systems [13]. As explained above, each OF has a special routing strategy.

- End-to-End Delay: Figure 3 shows the average latency from nodes to the root according to the number of hops between the nodes and the root. The latency is the amount of time it takes a frame to be transmitted from source to destination. As shown the NL-OF provides less latency than the OF0 and MRHOF-energy all the time while the MRHOFETX provides the same average of latency of NL-OF at 3 hops. OF0 provides more latency which confirms that the shortest path in term of number of hop does not necessarily minimize the delay.

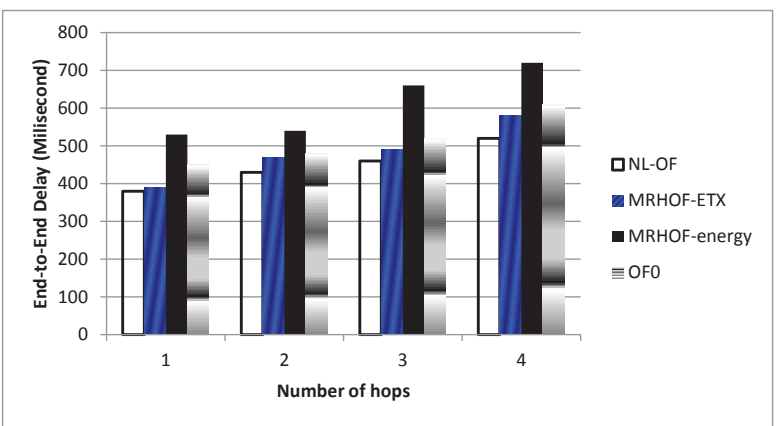

Fig. 3. The average End-to-End Delay as a function of the number of hops.

- Packet Loss: Figure 4 shows one of the main advantages of NL-OF. NL-OF guarantees a less amount of packet loss regarding to other OFs, which augments the reliability of the network. This result demonstrates the efficiency of NL-OF routing strategy for packet loss which severely degrades some applications. As the results show, NL-OF is always better than the other objective functions over time of the test. The network loses less number of packets when the OF is based on ETX because MRHOF-ETX chooses the next hop with minimum ETX which provides paths with low Packet Loss ratio. For the others OFs (OF0 and MRHOF-energy) the Packet Loss is higher due to the routing mechanism of these OF which optimize only the number of hops and the energy consumption respectively.

- Jitter: It is the amount of variation in latency time. In some applications Jitter degrades considerably the quality of network communications if it is so large. Figure 5 shows the amount of presence Jitter using NL-OF, MRHOF-ETX, MRHOF-energy and OF0 during simulation. It is clear that NL-OF assumes a lower Jitter comparing with others OFs $(\leq 80 \mathrm{~ms})$, except with 2 hops where NL-OF presents the larger Jitter. The advantage of NL-OF is that the NL-OF can provide routes with Jitter less or equal to the upper bound Jitter required by applications that are sensitive to QoS criteria.

- Power Consumption: To estimate the network lifetime with NL-OF, we have compared NL-OF with OF0, MRHOFETX and MRHOF-energy in term of Power Consumption 


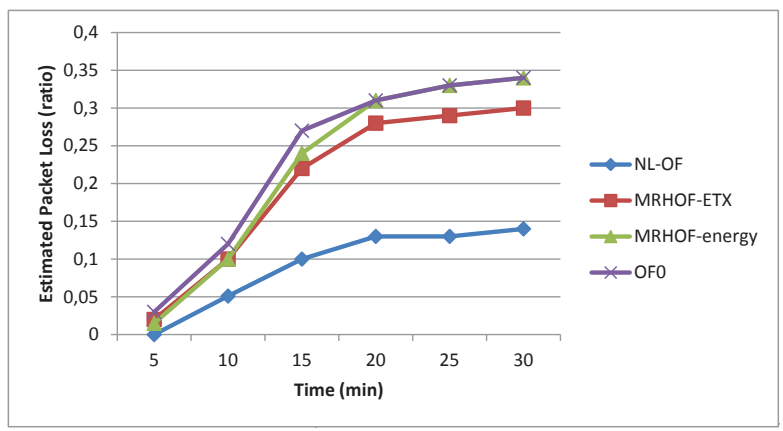

Fig. 4. Lost of packets over time.

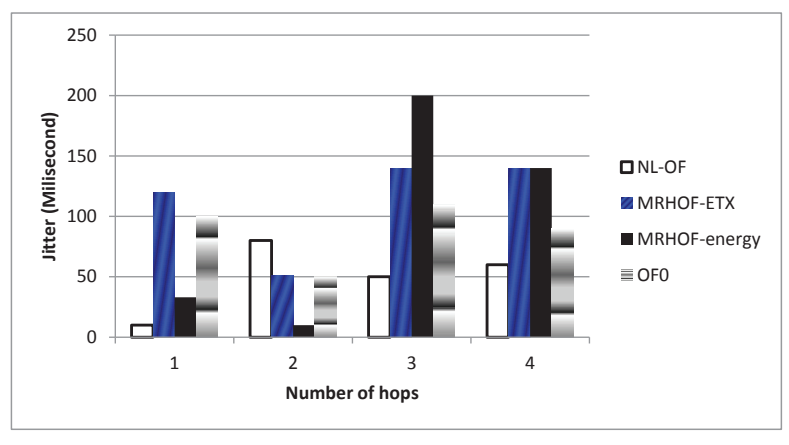

Fig. 5. Jitter in term of number of hops.

over time. Figure 6 presents the average power consumption. Trivially MRHOF-energy is the best in this challenge: 1.20 MW of energy was consumed through the 30 minutes of the test. MRHOF-ETX and OF0 consumed 1.5 MW when NL-OF consumed 1.4 MW. The consumption of energy was higher at the start of the experiment, because the DAG was not in a stable state (i.e. some nodes have not yet joined the DAG). Consequently, the Average Power Consumption is high. Since the 25th minute the network is more stable and the consumption of energy as well.

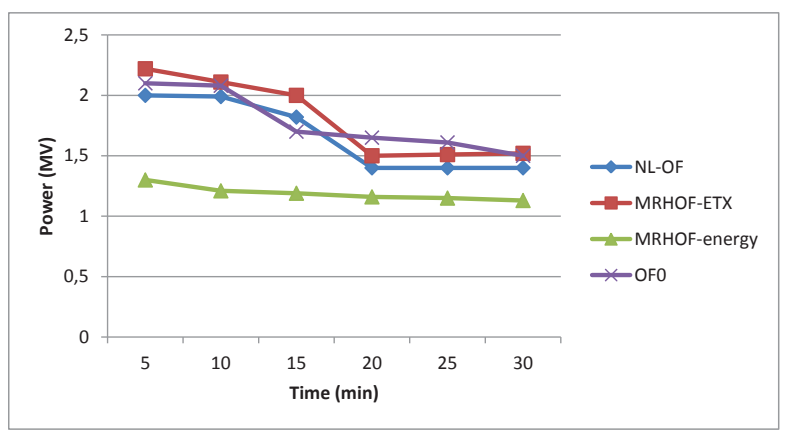

Fig. 6. Average Power Consumption over time.

\section{CONCLUSION}

We presented in this paper a new objective function, the Non-Linear Length OF (NL-OF) for improving the QoS in RPL. Our proposed OF performs with any number of metrics and constrains. On the theoretical part, we have argued that our greedy approach, although being approximate, fits better our setting while considering consider the memory constraints of LLNs devices. We have shown, based on experimental evaluation using Cooja, that our approach outperforms with respect to End-to-End Delay, Jitter, Packet Loss and Power Consumption. We observed that our approach performs better than others for Packet Loss.

As future work, NL-OF can be optimized while considering the routing cost. Also, we plan to study the structure of DAGs when using the exact solution.

\section{REFERENCES}

[1] T. Winter, P. Thubert, A. Brandt, J. Hui, R. Kelsey, P. Levis, K. Pister, R. Struik, J. Vasseur, and R. Alexander, RPL: IPv6 Routing Protocol for Low-Power and Lossy Networks. RFC 6550 \& (Proposed standard), Mar, 2012.

[2] P. Thubert, Objective Function Zero for the Routing Protocol for LowPower and Lossy Networks. RFC 6552 \& (Cisco Systems), Mar, 2012.

[3] JP. Vasseur, M. Kim, K. Pister, N. Dejean, and D. Barthel Routing Metrics Used for Path Calculation in LowPower and Lossy Networks. RFC 6551 \& (Cisco Systems), Mar, 2012

[4] M. Molnar, A. Bellabas and S. Lahoud. The cost optimal solution of the multi-constrained multicast routing problem. Computer Networks, 56(13):3136 - 3149, 2012

[5] H. Van Mieghem, P. De Neve and F. Kuipers. Hop-by-hop quality of service routing. Computer Networks, 37(34):407 - 423, 2001.

[6] F. Kuipers and P. Van Mieghem. MAMCRA: a constrained-based multicast routing algorithm. Computer Communications, 25(8):802 - 811, 2002.

[7] E.I Chong, S. Maddila and S. Morely. On finding signal-source singledestination k shortest paths. Computer Inf, 40 - 47, 1995.

[8] M.I Henig. The shortest path problem with two objective functions. European Journal of Operational Research, 25(2): 281 - 289, 1986.

[9] O. Gaddour, A. Kouba, N. Baccour and M. Abid OF-FL: QoS-aware fuzzy logic objective function for the RPL routing protocol. Modeling and Optimization in Mobile, Ad Hoc, and Wireless Networks (WiOpt): $365-372,2014$.

[10] O. Gnawali, P. Levis The ETX Objective Function for RPL. Internet Draft, Feb 2010.

[11] P. Karkazis, H.C. Leligou, P. Trakadas, T.H. Velivassaki. Design of primary and composite routing metrics for RPL-compliant Wireless Sensor Networ. International Conference on Telecommunications and Multimedia, 2012.

[12] Contiki Operating System. http://www.contiki-os.org/.

[13] K. Pister, P. Thubert, S. Dwars, P. Trakadas, T. Phinney. Industrial Routing Requirements in Low-Power and Lossy Networks. RFC 5673, Oct 2009.

[14] P. Levis, T. Clausen, J. Hui, O. Gnawali, J. Ko. The Trickle Algorithm. RFC 6209, Mar 2011. 